UNIVERSITAS, Volumen 4, Número 1, 2013, 29-32, ISSN 2071-2574

(C) UNAN-León, Editorial Universitaria

\title{
PRÁCTICAS PROFESIONALES, UNA CONTRIBUCIÓN A LA MEJORA DEL CURRÍCULUM EN LA CARRERA DE PSICOLOGÍA DE LA UNAN - LEÓN.
}

Olga V. Kulakova. Carrera de Psicología. Facultad de Ciencias Médicas. Universidad Nacional Autónoma de Nicaragua (UNAN- León). Nicaragua.

\section{RESUMEN}

Antecedentes y objetivos. Las Prácticas Profesionales representan una experiencia valiosa e importante en la formación de los estudiantes universitarios. Sin embargo existe poca documentación sobre la evaluación de esta área de formación. Tomando como contexto el re-diseño curricular, con el fin de adecuar los planes de estudio al Modelo de Currículum Basado en Competencias, como objetivos se planteó analizar los escenarios y actividades de aprendizaje de las Prácticas Profesionales (PP) de la carrera de Psicología e identificar las necesidades formativas percibidas por los estudiantes. Metodología. Se realizó un estudio cuanticualitativo aplicando una encuesta semi-estructurada a los estudiantes del último año de la carrera de Psicología. Además, se realizaron entrevistas a las autoridades y a los docentes que han tenido a cargo las tutorías y las supervisiones. Resultados. Predominaron las preocupaciones relacionadas con una débil estructura metodológica y organizativa de esta área curricular, una falta del vínculo entre teoría y práctica y una falta de diversidad de contextos para la realización de las prácticas. Conclusiones. A pesar que las PP son vistas como una oportunidad para que el estudiante desarrolle sus competencias en las condiciones reales, la planificación actual de las PP en la carrera de Psicología limita el desarrollo de las capacidades para actuar en distintos niveles de contacto y en diferentes ámbitos de la profesión.

Palabras Clave: Carrera de Psicología. Prácticas Profesionales. Currículum

\section{INTRODUCCIÓN.}

Una de las valiosas e importantes experiencias para los estudiantes universitarios, desde el punto de vista de su formación profesional, son las prácticas profesionales $(\mathrm{PP}) .{ }^{(1,2)}$ Desde que se inicia la Reforma Curricular en la UNAN - León, en 1996, las PP, son incorporadas a los planes de estudio de todas las carreras y valoradas en todas sus dimensiones, adoptando la concepción y los planteamientos teóricos del modelo del Currículum Integral de Walter Peñaloza en el cual se insiste en desarrollar las PP de forma graduada, según sus niveles de complejidad.

Las PPen la carrera de Psicología de la Facultad Ciencias Médicas comenzaron a figurar en el Plan de Estudio de 2003, incorporando a los estudiantes en los centros educativos, ONGs, hospitales, centros de salud y de Atención Psicosocial (CAPS) desarrollando actividades prácticas a "Nivel de Familiarización".(3)
En el año 2004, se comenzó a realizar otros esfuerzos para organizar actividades prácticas dentro de las asignaturas Psicopatología y en el año 2005, en la asignatura Psicología Forense.

Sin embargo, con la planificación actual de las PP en la carrera de Psicología se percibe un clima de insatisfacción entre los estudiantes y preocupación entre algunos docentes.

Las primeras manifestaciones de esta insatisfacción fueron identificadas tras una investigación realizada por un grupo de profesores en el año 2002 en el cual los estudiantes encuestados de III, IV y V años de la Carrera reflejaron que existía discrepancia entre el perfil profesional establecido por la Carrera y preparación práctica orientada, principalmente, al ámbito clínico de la profesión. ${ }^{(4)}$

Desde el año 2006, en la UNAN - León se comenzó a desarrollar nuevas políticas y 
estrategias para re-introducir el Sistema de Créditos Académicos (SCA) como forma de organización y de estructuración de los planes de estudio de todas las carreras que se ofrecen ${ }^{(2)}$.

En este contexto del re-diseño curricular y en sentido de mejora. Se planteó analizar los escenarios y actividades de aprendizaje de las PP e identificar las necesidades formativas percibidas por los estudiantes de la carrera de Psicología de la UNAN - León.

\section{MATERIAL Y MÉTODOS.}

Usando un abordaje cuanti-cualitativo, se realizó un diagnóstico situacional sobre los aspectos organizativos, los escenarios y actividades de aprendizaje de las PP de Psicología, se valoró la opinión sobre las necesidades formativas percibidas $y$ sugerencias para mejorar esta área curricular.

En el estudio participaron 42 estudiantes matriculados en el último año de la Carrera, que cursaron las PP-I y las PP-II en el período 2007-2008. También participaron cinco de los seis docentes que han tenido a cargo las tutorías y las supervisiones en los dos períodos de estas PP y las autoridades (director de la carrera, vice-decana de la facultad).

La recolección de datos de los estudiantes se realizó a través de una encuesta de opiniones auto-administrada y de los docentes y autoridades a través de una entrevista con tópicos abiertos.

\section{RESULTADOS Y DISCUSIÓN.}

Los resultados de este estudio ponen en evidencia que el proceso actual de la formación práctica en la carrera de Psicología está hoy día debilitado. Las PP están deficitarias en cuanto a la estructura metodológica y organizativa, los niveles de contacto con diferentes ámbitos de actuación y la diversidad de contextos.
Según los resultados obtenidos, en las PPI, el 100\% de los estudiantes ejercieron sus prácticas en los Centros Escolares (ámbito educativo) Sin embargo, en las PP-II, la rotación por los ámbitos de la profesión fue parcial y los estudiantes no obtuvieron las mismas oportunidades de la práctica: el 47\% ejercieron en ámbito clínico, el $33 \%$ en el ámbito psicosocial y el $20 \%$ se vieron obligados ejercer las mismas prácticas educativas.

Distribución porcentual de sugerencias realizadas por estudiantes $(n=42)$

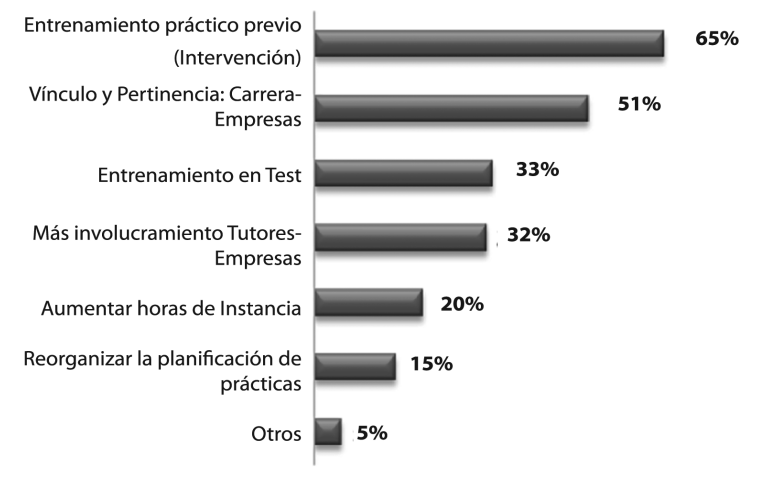

Es indispensable que los estudiantes en las PP tengan las mismas oportunidades de formación y cumplan con la rotación por los cuatro ámbitos de actuación profesional. El perfil académico-profesional de la carrera así lo establece: "el estudiante egresado de la carrera de Psicología será capaz de brindar atención integral al individuo, familia y comunidad en el campo clínico, educativo, laboral y socialcomunitario mediante acciones de promoción, prevención, intervención y rehabilitación psicológica." $(3,5)$

En cuanto a las actividades realizadas, los estudiantes manifestaron problemas relacionados con la forma en que los administrativos o profesionales de los centros de prácticas entienden la inserción de los practicantes. Más de la mitad de los estudiantes (56\% en las PP-I y $43 \%$ en las PP-II) denunciaron realizar actividades no 
relacionadas con el quehacer del psicólogo o, a veces, llegar a "no hacer nada". Se les ha dado pocas opciones de participación, se ha aprovechado la presencia de estudiantes para cubrirtrabajospendientes detipoadministrativo o, como ha ocurrido en los centros escolares, cubrir la baja de algún profesor del centro o las faltas en la planificación y seguimiento de los alumnos en el aula.

Otro aspecto se refiere al tipo de tareas que realizaron los estudiantes durante sus prácticas.

Al calcular la contingencia de las tareas realizadas en las dos prácticas, se obtuvo que el $100 \%$ de los estudiantes lograron realizar entrevistas psicológicas, un $89 \%$ realizaron charlas. Muy pocos estudiantes (12\%) lograron aplicar algunas pruebas psicológicas y/o intervenir con el usuario y un 59\% de los estudiantes no lograron la aplicación de las pruebas, ni intervención en ningún semestre de las prácticas. De todos los estudiantes un $65 \%$ se dedicaron a las tareas no relacionadas con el perfil profesional.

Al analizar las competencias que deberían demostrar los egresados de Psicología ${ }^{(3,5,)}$, nos enfrentamos a una realidad preocupante: una parte sustancial de las competencias específicas planteadas, que son indispensables para una formación profesional de calidad, no son factibles debido a que las tareas que realizan los estudiantes son limitadas. Muchas de estas competencias necesitan previa experimentación obligatoriamente en situaciones y contextos reales.

Los problemas derivados de la organización más mencionados por los estudiantes $(86 \%)$ y los tutores $(80 \%)$ son: la selección inadecuada de los centros o instituciones para realizar las prácticas. Para crear un verdadero vínculo entre la universidad y los centros de las prácticas, es necesario crear un diálogo respecto a qué estamos entendiendo por formación práctica del psicólogo, qué papel tiene la universidad y las instituciones colaboradoras en el proceso de la formación práctica, cuáles son las responsabilidades y los compromisos que se asumen en la construcción del aprendizaje de futuros egresados.

En relación a los niveles que gradúan la formación práctica, todos los docentes-tutores opinaron que los estudiantes de psicología solamente desarrollan sus prácticas a nivel de "Familiarización" y en algunos casos, quedan a nivel de "Sensibilización".

Es indispensable plantear las prácticas por niveles de progresividad y complejidad (3, 6, 7). Según Peñaloza (1998), para formar profesionales en el pleno sentido de la palabra se necesita no solo una secuencia de asignaturas, sino también una secuencia de PP, a lo largo de los semestres de cada carrera: Nivel Inicial, Intermedio y Terminal.

En cuanto a las necesidades formativas percibidas por los estudiantes, en gran parte, las respuestas aludieron en términos generales a la necesidad de que se prepare a los estudiantes para la actuación práctica que han de desempeñar en los centros.

Predominó la demanda de conocimientos procedimentales $(68 \%)$, relativos al cómo realizar determinadas acciones en el momento de evaluación e intervención psicológica en diferentes ámbitos del ejercicio profesional. Un 65\% demandaron un "entrenamiento previo" en estrategias y técnicas de intervención, como psicoterapias, y un $33 \%$ en técnicas de evaluación, como aplicación de pruebas psicológicas o Test, en todos los ámbitos del ejercicio profesional, antes de la inserción en los centros donde realizan las prácticas. 


\section{CONCLUSIONES.}

Las PP son vistas como una oportunidad para que el estudiante desarrolle sus competencias en las condiciones sin embargo la planificación actual de las PP en la carrera limita el desarrollo de las capacidades para actuar en distintos niveles de contacto y en diferentes ámbitos de la profesión.

Las necesidades de formación expresadas por los estudiantes sugieren aspectos importantes de mejora. Uno de ellos es asumir y desarrollar las PP en diferentes niveles (inicial, intermedio y terminal), incluyendo en su diseño la presencia y experimentación equitativa de los principales campos de desempeño del psicólogo. Otro aspecto se refiere a los planteamientos curriculares del propio plan de formación del psicólogo, en el cual se requiere fortalecer la interrelación entre las PP y otras áreas de conocimiento implicadas, así como establecer un verdadero vínculo entre el binomio teoría-práctica.

Ante esta nueva configuración curricular de las PP, es importante que la carrera de Psicología incremente su compromiso en la priorización de la gestión en esta área curricular.

\section{AGRADECIMIENTOS.}

A los estudiantes de Psicología, de la promoción 2009, por su participación en el estudio y deseo de colaborar para las mejoras de la carrera de Psicología, a los docentes y autoridades por compartir su sincera opinión.

\section{REFERENCIAS.}

1. Marco Referencial para la Reforma Curricular en la UNAN - León (1998) Comisión de la Reforma Curricular, Vice-rectoría Académica, Universidad Nacional Autónoma de Nicaragua, UNAN - León.
2. Marco Referencial para el Diseño Curricular por Competencias (2007), Comisión Curricular, Vice-rectoría Académica, Universidad Nacional Autónoma de Nicaragua UNAN - León.

3. Macroprogramación de la Carrera de Psicología (2006), Facultad Ciencias Médicas. Universidad Nacional Autónoma de Nicaragua, UNAN León.

4. Rodríguez-Madariaga, A., Romero I., Gómez H., (2002). "Macro-programación de la Carrera de Psicología", Tesis de Grado, Maestría en Educación Superior en Salud, Facultad Ciencias Médicas, Universidad Nacional Autónoma de Nicaragua, UNAN - León. 14 -15.

5. Rodríguez-Cruz, J., Rodríguez-Madariaga, A. y Kulakova, O. (2007). Macroprogramación del Plan de Estudio de la Carrera de Psicología del Diseño Curricular por Competencias y Basado en el Sistema de Créditos Académicos. Facultad Ciencias Médicas, Universidad Nacional Autónoma de Nicaragua, UNAN - León. p.62.

6. Medina, E., Valladares, W., Sánchez, H. y Saldaña, J. (1997). Proyecto Educativo de la UNAN - León. Universidad Nacional Autónoma de Nicaragua, UNAN - León

7. Peñaloza, W. (1996). "Diseño Curricular: Profesión y Prácticas Profesionales". Curso de la Escuela de Verano, Consejo Nacional de Universidades - CNU, Managua, Nicaragua. 